\title{
Influence of annealing temperature on the structural and magnetic properties of $\mathrm{FeGaSiB}$ thin films
}

\author{
Qayes A. Abbas ${ }^{\mathrm{a}, \mathrm{b}}$, Michelle Rodrigues ${ }^{\mathrm{a}}$, Saturi Baco ${ }^{\mathrm{a}}$, Shaoliang Guan ${ }^{\mathrm{c}, \mathrm{d}}$, Nicola A Morley ${ }^{\mathrm{a}, *}$ \\ ${ }^{a}$ Department of Materials Science and Engineering, University of Sheffield, Sheffield S1 3JD, UK \\ ${ }^{\mathrm{b}}$ Department of Physics, College of Science, University of Anbar, Anbar, Iraq \\ ' School of Chemistry, Cardiff University, Cardiff CF10 3AT, UK \\ ${ }^{\mathrm{d}}$ HarwellXPS, Research Complex at Harwell, Rutherford Appleton Laboratory, Didcot OX11 OFA, UK
}

A R T I C L E I N F O

\section{Keywords:}

Thin magnetic films

Amorphous

Heat treatment

\begin{abstract}
A B S T R A C T
Understanding the relationship between the structure and magnetic properties of soft magnetic thin films is important in the development of magnetic sensors and electronic devices. This work has systematically studied how the annealing temperature changes the morphology and hence the magnetic properties of $60 \mathrm{~nm} \mathrm{FeGaSiB}$ thin films. The as-grown and $100{ }^{\circ} \mathrm{C}$ annealed films were amorphous/nanocrystalline with soft magnetic properties. As the annealing temperature increased, so did the coercive field of the films, which was found to be due to the formation of polycrystalline grains, with random texture. The grain size increased with increasing annealing temperature, while the Curie Temperature and magnetostriction constant decreased. For the saturation induction, there was a large decrease between the $100{ }^{\circ} \mathrm{C}$ annealed film and the $250{ }^{\circ} \mathrm{C}$ annealed film, due to the film morphology changing from amorphous to polycrystalline. Thus there is strong correlation between the crystallinity and the soft magnetic behaviour of FeGaSiB films.
\end{abstract}

\section{Introduction}

Soft magnet thin films are used in a wide range of applications including microelectronic mechanical system (MEMS) devices [1-3]. The main focus is to fabricate films, which have the optimum properties for the MEMS application they are going to be used in. For example, if the films are going to be used as microinductors then the ideal properties are coercive field $\left(H_{c}\right)<10 \mathrm{~A} / \mathrm{m}$, saturation magnetisation $\left(M_{s}\right)>800 \mathrm{kA} / \mathrm{m}$ and magnetostriction constant $\left(\lambda_{s}\right)<5 \mathrm{ppm}[2,4]$, while if they are going to be used as strain sensors, $H_{c}<1 \mathrm{kA} / \mathrm{m}$, $M_{s}>800 \mathrm{kA} / \mathrm{m}$ and $\lambda_{s}>50 \mathrm{ppm}$ [5]. This means understanding how the films' morphology influences the magnetic properties, such as coercive field, saturation magnetisation, damping constant, resistivity and magnetostriction constant is extremely important.

Previous work on soft magnetic alloys [6], has found that the coercive field is strongly linked to the sample's grain size, such that for grain sizes less than the magnetocrystalline exchange length, the coercive field increase is proportional to the grain size to the power six $\left(D^{6}\right)$. This means to achieve small coercive fields either nanocrystalline grains or amorphous materials are required, due to the absence of grains and grain boundaries. This holds true for bulk and ribbon soft magnetic alloys, as it is associated with Bloch domain walls forming.
For thin films below a certain thickness, Neel domain walls are more likely to form rather than Bloch walls. For Neel walls the magnetostatic exchange length is more important than the magnetocrystalline exchange length [7]. Therefore, investigation into the soft magnetic properties of thin film as a function of the morphology is important.

The majority of the research on amorphous magnetic films has focused on FeSiB. Sun et al. [8] studied how the magnetic properties and domain structure of $\mathrm{FeSiB}$ films, were affected by annealing at $540{ }^{\circ} \mathrm{C}$ for $1 \mathrm{~h}$. They found that the as-grown $100 \mathrm{~nm}$ FeSiB films consisted of an amorphous matrix with $\alpha$-Fe crystallites, which grew during the annealing process. The as-deposited films had a magnetostriction constant of $6.5 \mathrm{ppm}$, which reduced to $0.45 \mathrm{ppm}$ after annealing, while the coercive field increased to $\sim 1.4 \mathrm{kA} / \mathrm{m}$ for the annealed film. While Neagu et al. [9] grew $300 \mathrm{~nm}$ FeSiB films on glass, and found that the coercive field of the as-grown film was $350 \mathrm{~A} / \mathrm{m}$, which was reduced to $23 \mathrm{~A} / \mathrm{m}$ after annealing for $1 \mathrm{~h}$ at $390{ }^{\circ} \mathrm{C}$. They state this was due to surface relaxation, no structural data of the annealed films was given.

FeGa thin films [10-12] have some of the highest magnetostriction constants (up to $100 \mathrm{ppm}$ ) for thin films, but also have large coercive fields $(>20 \mathrm{kA} / \mathrm{m})$, thus work by Abbas et al. [13,14] investigated adding $\mathrm{Ga}$ into $\mathrm{FeSiB}$, to fabricate films with high magnetostriction constants and small coercive fields. They found that the magnetic

\footnotetext{
* Corresponding author.

E-mail address: n.a.morley@sheffield.ac.uk (N.A. Morley).
} 
properties of FeGaSiB films strongly depended upon the fabrication parameters, with the highest magnetostriction constant $(\sim 18 \mathrm{ppm})$ and lowest coercive field $\sim 0.3 \mathrm{kA} / \mathrm{m}$ being achieved at low pressures $(<0.5 \mathrm{~Pa})$ and powers $(<30 \mathrm{~W})$.

This paper investigates the structural and magnetic properties of a series of $60 \mathrm{~nm} \mathrm{FeGaSiB}$ thin films, which have undergone different annealing temperatures, to determine how changing the morphology of the films changes the magnetic properties.

\section{Experimental procedure}

The films were grown on Si (100) substrates with the native oxide layer in place. The substrates' dimensions were $10 \mathrm{~mm} \times 15 \mathrm{~mm}$. They were cleaned using acetone and isopropyl alcohol (IPA) before growth. To allow for a measurement of the film thickness, a small spot of polymethyl methacrylate (PMMA) was placed on the corner of the silicon before growth. The fabrication took place in a co-sputteringevaporation deposition system [11]. Before growth the chamber was pumped down to $5 \times 10^{-5} \mathrm{~Pa}$ and left at base pressure overnight. To achieve the co-sputtering-evaporation, the Ga was evaporated simultaneously with the FeSiB being sputtered. The Ga was purchased from Goodfellow with a purity of $99.99 \%$. The $\mathrm{Fe}_{85} \mathrm{Si}_{10} \mathrm{~B}_{5}$ target foil was purchased from Metglas, Inc. The initial procedure involves heating the $\mathrm{Ga}$ up in a high temperature crucible and monitoring the Ga flux rate on a quartz rate monitor. The Ga flux rate is kept constant, but due to the low melting point of $\mathrm{Ga}$, it is not possible to calibrate the rate in terms of thickness, thus the Ga rate is given in arbitrary units (a.u). Once the Ga rate is constant (for these films at 0.2 a.u.), Argon gas is added to the chamber at a pressure of $0.4 \mathrm{~Pa}$. A power of $20 \mathrm{~W}$ is applied to the FeSiB target and a plasma is struck. Before the film growth, the FeSiB target is "pre-sputtered" for $10 \mathrm{~min}$ to remove any impurities or oxide layer from the surface. Once the target has been cleaned, the substrate shutter is opened, and the film growth takes place. The film thickness is monitored on a second calibrated thickness monitor next to the substrate within the growth chamber. Once the required thickness has been reached the substrate shutter is automatically closed. All the films were grown at the room temperature within the system. Please refer to [11] for further details on this co-sputtering-evaporation deposition system.

After growth, the films were annealed at different temperatures (100, 250, 350 and $400{ }^{\circ} \mathrm{C}$ ) under vacuum for $30 \mathrm{~min}$, within the growth chamber. From literature [15], the transition from amorphous to crystalline films for FeSi occurred between $180{ }^{\circ} \mathrm{C}$ and $250{ }^{\circ} \mathrm{C}$, when annealed for $30 \mathrm{~min}$ under vacuum. Thus for this research, the annealing time was chosen to be the same, and a range of temperatures, which spanned this transition region was chosen. Five films were studied for this work, these were: as-deposited (As-FGSB), i.e. no annealing and heated treated at the different temperatures (100-FGSB, 250-FGSB, $350-$ FGSB and 400-FGSB). These growth conditions previously gave the as-grown composition of the films to be $\mathrm{Fe}_{82} \mathrm{Ga}_{7} \mathrm{Si}_{5} \mathrm{~B}_{6}$ [13]. After growth and annealing, the films were cleaned using acetone, to remove the PMMA from the surface, and leave a sharp edge between the substrate and the film. Using an atomic force microscope (AFM), the thickness of each film was determined to be $60 \pm 1 \mathrm{~nm}$, which was in agreement with the calibrated thickness monitor in the deposition chamber.

A range of different characterisation techniques were used to fully understand the films. Small angle x-ray diffraction (XRD) was used to investigate the morphology of the films. A Siemens D5000 (Cu, GA$\mathrm{XRD}$ ) was used in glancing angle mode. $\mathrm{Cu} \mathrm{K}_{\alpha 1}$ radiation was used to scan the samples with a wavelength of $1.5418 \AA$ with the X-Ray tube working at $40 \mathrm{kV}, 40 \mathrm{~mA}$. The $2 \theta$ value range was measured from 20 to $80^{\circ}$, with a step size of $0.020^{\circ}$, a glancing angle of $1.0^{\circ}$ and a wait time of $7 \mathrm{~s}$. From the data, the lattice constant was determined from Bragg's law and the minimum grain size from Scherrer equation [16]. An AFM in tapping mode was used to determine the surface roughness of the films, as this can be linked to the morphology, and the grain size. X-ray photoelectron spectroscopy (XPS) was used to determine the composition of the films. It was performed on a Thermo Fisher Scientific NEXSA spectrometer. Samples were analysed using a micro-focused monochromatic Al x-ray source (19.2 W) over an area of approximately 100 microns. Data were recorded at pass energies of $150 \mathrm{eV}$ for survey scans and $40 \mathrm{eV}$ for high resolution scan with $1 \mathrm{eV}$ and $0.1 \mathrm{eV}$ step sizes respectively. Charge neutralisation of the sample was achieved using a combination of both low energy electrons and argon ions. The measurements were taken on the film surface only.

Magneto-optic Kerr effect (MOKE) magnetometry was used to measure the normalised magnetisation loops in the plane of the film. The magnetic field was applied along different in-plane directions with respect to a designated $0^{\circ}$ direction. From these loops, the anisotropy could be inferred, with the magnetic parameters (anisotropy field, coercive field and remnant magnetisation) being determined. A MPSM3 in VSM mode was used to determine the saturation magnetisation of the films at $300 \mathrm{~K}$. Due to the small moment of the films, the silicon substrate magnetic moment was subtracted from the VSM data, to determine the magnetic moment of each film. As the heat treatment for these films was for a short period of time, and even this changed the morphology of the films, measurements above $50{ }^{\circ} \mathrm{C}$ were not carried out in case they changed the sample properties during the measurement. Thus magnetisation $(M)$ vs temperature $(T)$ curves were taken between $-271{ }^{\circ} \mathrm{C}$ and $50{ }^{\circ} \mathrm{C}$, and the Curie Temperature $\left(T_{c}\right)$ extrapolated from the M-T curves using the empirical Bloch law [17]:

$\frac{M(T)}{M(0)}=\left(1-\left(\frac{T}{T_{c}}\right)^{3 / 2}\right)^{\beta}$

where $M(0)$ is the magnetisation at $0 \mathrm{~K}$, and $\beta$ is a constant. The magnetisation was also determined for the as-FGSB and 100-FGSB films using a vector network analyser ferromagnetic resonance (VNA-FMR) system, operating in a 1-port (reflection) mode. The saturation magnetisation values were extracted by fits to the Kittel equation, see [18] for further details. It was not possible to measure the other films using the VNA-FMR, as their coercive fields were too large for the applied field, which meant it was not possible to fit the Kittel equation to the data.

The magnetostriction constant was determined using the Villari Method, which is a mechanical method involving bending the films over known a set of known bend radii $(R=500 \mathrm{~mm}, 400 \mathrm{~mm}$ and $300 \mathrm{~mm}$ ). The normalised magnetisation hysteresis loop was measured on the MOKE magnetometer. From each loop, the anisotropy field $\left(H_{k}\right)$ was determined and plotted against the inverse bend radius $[16,19]$. The magnetostriction constant was then calculated from:

$\lambda=\left(\frac{d H_{k}}{d \frac{1}{R}}\right) \frac{2 \mu_{o} M_{s}\left(1-v_{s}^{2}\right)}{3 \tau_{s} Y_{s}}$

Where $M_{s}$ is the saturation magnetisation of the film, $Y_{s}$ is the Young's Modulus of the substrate, $\tau_{s}$ is the substrate thickness, and $\nu_{s}$ is the Poisson ratio of the substrate. Care is taken during the experiment not to snap the films, when applying the strain.

\section{Results and discussion}

The composition of each annealed films was determined using XPS, as this is a surface technique, and therefore will not detect the Si substrate, as other techniques such as energy dispersive $\mathrm{x}$-ray spectroscopy (EDS) are deeper depth probes, which will also detect the Si substrate. Previous work found that XPS was the most reliable method for determining the composition of FeGaSiB films [14]. From table 1, it is observed that the as-FGSB film has a much higher Fe content compared to the other films, which is also higher than those measured previously for the same growth technique [14]. One reason could be due to the fact that XPS is a surface technique, and there is a concentration gradient 
Table 1

Composition of the FeGaSiB films determined from the XPS data

\begin{tabular}{lllll}
\hline Film & $\begin{array}{l}\text { Fe (conc\%) } \\
( \pm 1)\end{array}$ & Ga (conc\%) $( \pm 1)$ & Si (conc\%) $( \pm 1)$ & B(conc\%) $( \pm 1)$ \\
\hline As-FGSB & 93 & 2 & 3 & 2 \\
$100-F G S B$ & 85 & 4 & 6 & 5 \\
$250-F G S B$ & 82 & 4 & 11 & 3 \\
$350-F G S B$ & 82 & 4 & 12 & 2 \\
$400-F G S B$ & 70 & 4 & 24 & 2 \\
\hline
\end{tabular}

through the film. The Ga and B concentrations are similar to those in the annealed films, with only the Si concentration being smaller than expected. The $\mathrm{Fe}\left(2 \mathrm{p}_{3 / 2}\right)$ peak (Fig. 1a) for the as-FGSB film has a smaller Fe peak at $706.7 \mathrm{eV}$ and a larger $\mathrm{Fe}_{2} \mathrm{O}_{3}$ peak at $710.8 \mathrm{eV}$ compared to the annealed films. Fe-based films normally have a $\sim 2 \mathrm{~nm}$ native oxide layer when grown [20]. The XPS data suggests that this layer was thicker for the as-FGSB film compared to the annealed films, this could then explain the higher Fe content compared to the other elements. Fig. 1b shows the XPS spectra of the films for the $\mathrm{B}(1 \mathrm{~s}), \mathrm{Ga}$ (3s) and $\mathrm{Si}(2 \mathrm{~s})$ binding energies. It is observed that the area of the $\mathrm{B}$ peaks decreases with annealing temperature and the Ga peaks area is roughly constant, while there is a large increase in the Si peak, associated with the $\mathrm{Si}$ diffusion. All the films have a lower Ga concentration compared to the expected composition [13]. Thus for the 100-FGSB, 250-FGSB and 350-FGSB films, the Ga content within the films is roughly constant, with the $\mathrm{Fe}$ and $\mathrm{B}$ content decreasing. While the $\mathrm{Si}$ concentration increases with increasing annealing temperature. The
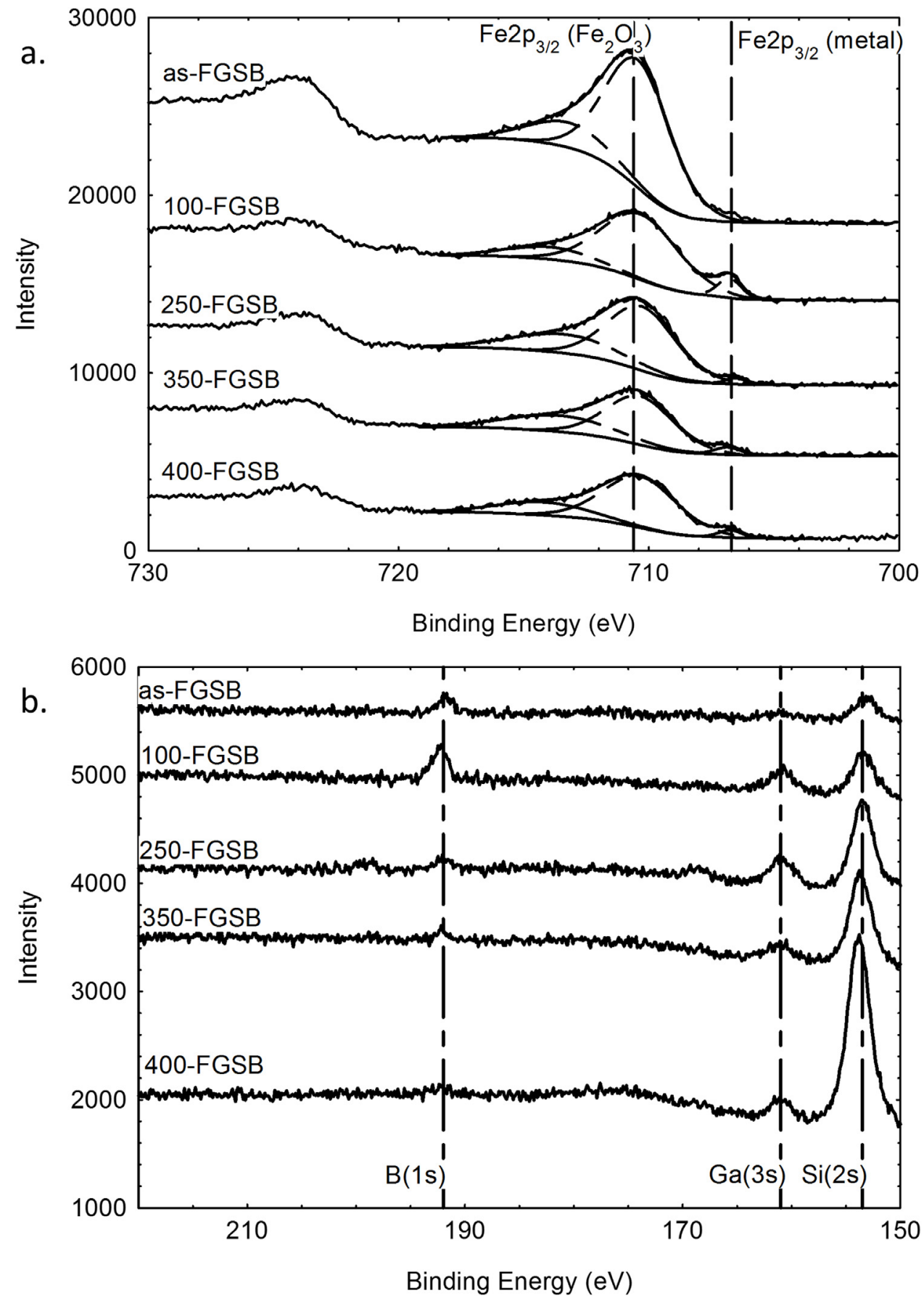

Fig. 1. XPS spectra of the (a). Fe(2p) and (b). B(1s), Ga(3s) and Si(2s) binding energies. The dashed lines give the binding energies for the different elements. 

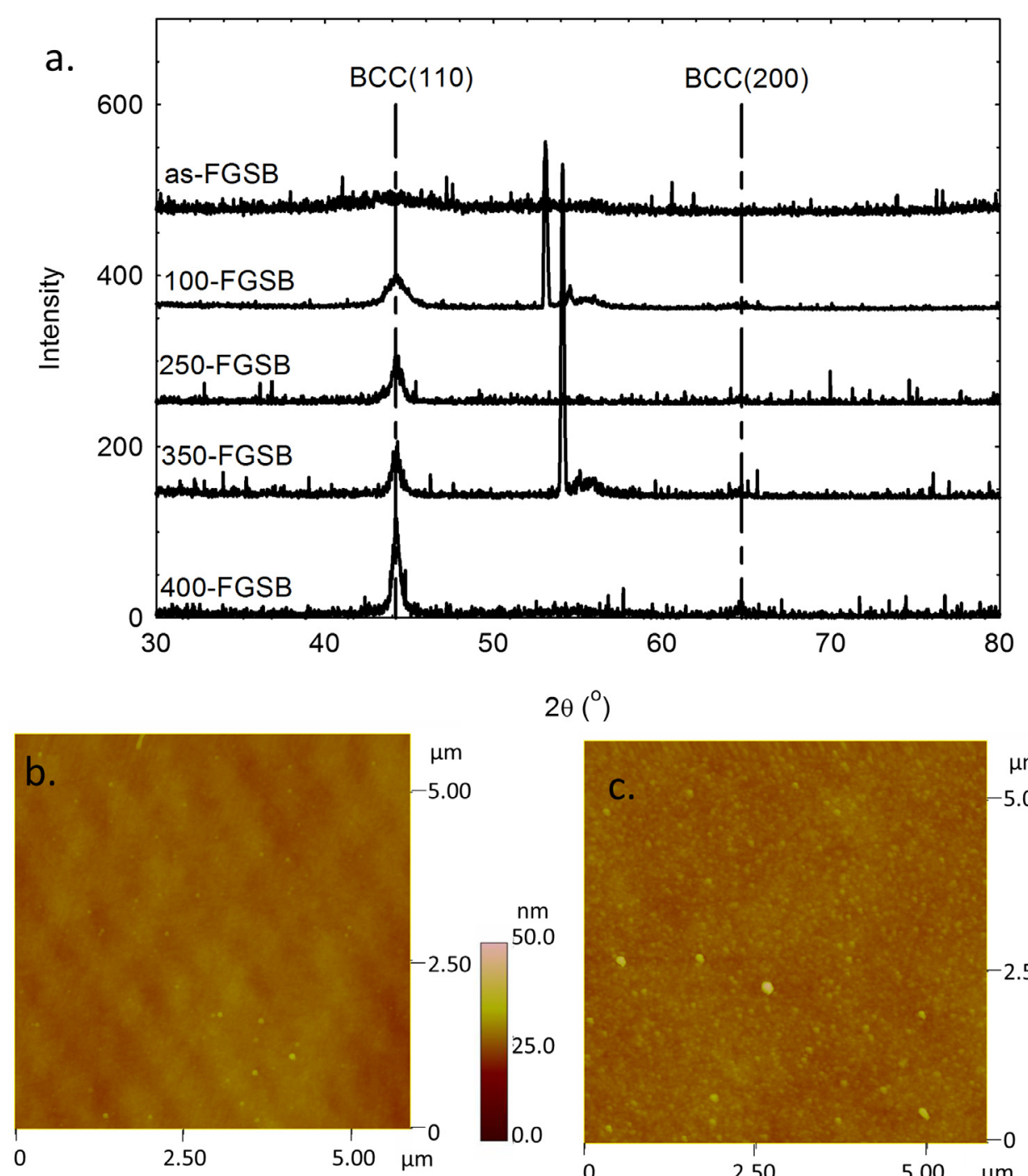

$2 \theta\left({ }^{\circ}\right)$
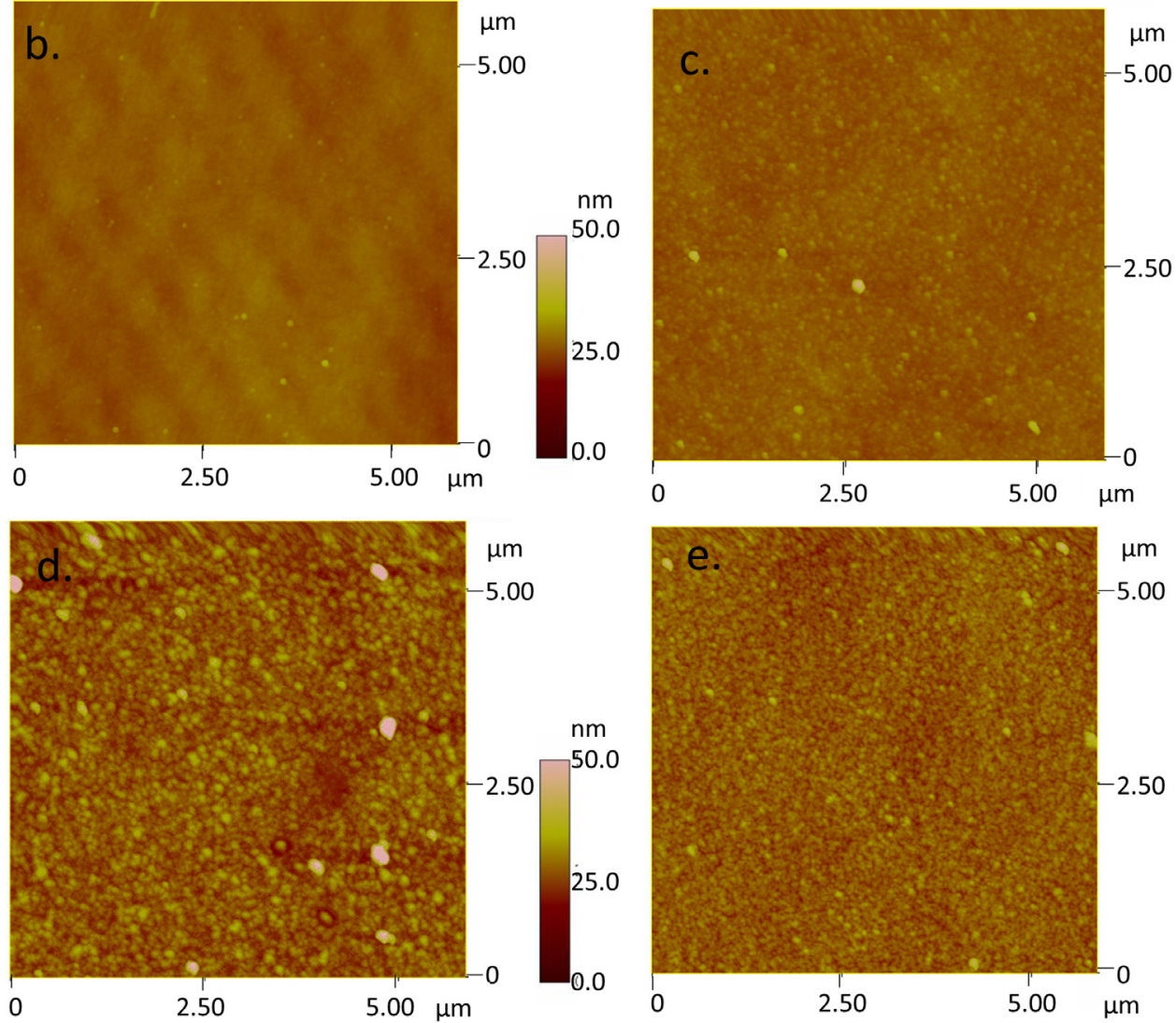

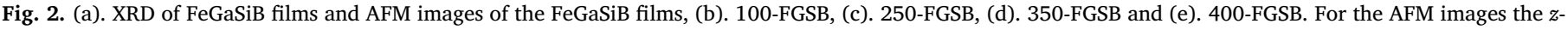
direction scale bar is the same for all the films.

initial increase would seem to be at the expense of the B and Fe. For the 400-FGSB film, the Si concentration is double that of the 350-FGSB film, and is at the expense of the Fe.

Previous work on FeSi films on Si silicon substrates have studied the diffusion of $\mathrm{Fe}$ and $\mathrm{Si}$ across the interface as a function of annealing temperature. Isobe et al. [21] determined the diffusion coefficient of Fe into $\mathrm{Si}$ substrate as a function of annealing temperature. Using their equation for the diffusion coefficient, $D_{F e}$ :
$D_{F e}=9.5 e^{-4} \exp \left(\frac{-0.65}{k T}\right)$

Where $k$ is the Boltzmann constant (units: $\mathrm{eV} / \mathrm{K}$ ) and $T$ is the annealing temperature, the diffusion length of $\mathrm{Fe}$ into silicon can be determined for an annealing time of $30 \mathrm{~min}$ for different annealing temperatures. It is found that even at low annealing temperatures, Fe will diffuse into the silicon substrate (diffusion length $\sim 45 \mathrm{~nm}$ ), while increasing the annealing temperature to above $\sim 200{ }^{\circ} \mathrm{C}$ increases the diffusion 
Table 2

Structural properties of the FeGaSiB films

\begin{tabular}{lllll}
\hline Film & Lattice Constant $(\AA)$ & $\begin{array}{l}\text { Crystallite } \\
\text { size (XRD) } \\
(\mathrm{nm})\end{array}$ & $\begin{array}{l}\text { Surface } \\
\text { Roughness } \\
(\mathrm{nm})\end{array}$ & $\begin{array}{l}\text { Grain size } \\
(\mathrm{AFM})(\mathrm{nm})\end{array}$ \\
\hline As-FGSB & - & $<2$ & $0.8 \pm 0.4$ & - \\
100-FGSB & $2.89 \pm 0.04$ & $6 \pm 3$ & $0.9 \pm 0.7$ & - \\
250-FGSB & $2.893 \pm 0.008$ & $9 \pm 3$ & $1.2 \pm 0.8$ & $110 \pm 10$ \\
350-FGSB & $2.893 \pm 0.008$ & $12 \pm 3$ & $3.5 \pm 2.0$ & $160 \pm 10$ \\
$400-F G S B$ & $2.890 \pm 0.008$ & $14 \pm 3$ & $2.0 \pm 1.0$ & $130 \pm 10$ \\
\hline
\end{tabular}

coefficient and length, i.e. at $425{ }^{\circ} \mathrm{C}$ the diffusion length is $\sim 50 \mu \mathrm{m}$. As the $\mathrm{FeGaSiB}$ films were grown on Si substrates with the native oxide in place, this very thin oxide layer will act as a barrier to diffusion below an activation energy. From previous literature on $\mathrm{Fe} / \mathrm{Si}$ multilayers and Fe films on silicon, at temperatures above $200{ }^{\circ} \mathrm{C}, \mathrm{Si}$ from the substrate can diffusion across the interface into the Fe film and vice versa [22, 23]. It would seem in the $\mathrm{FeGaSiB}$ films that the initial diffusion occurred after $250{ }^{\circ} \mathrm{C}$ and accelerated above $350{ }^{\circ} \mathrm{C}$. Suggesting that the activation energy for diffusion across the oxide layer is above $200{ }^{\circ} \mathrm{C}$.

From the XRD data in Fig. 2, it is observed that the as-FGSB film has a broad amorphous peak at around $\sim 44^{\circ}$, and as the annealing temperature increases, so this peak becomes sharper, with a second peak appearing at $\sim 64^{\circ}$. These peaks are associated with the BCC (110) and (200) directions. The signal to noise ratio (SNR) is larger for the asFGSB film compared to the annealed films. This probably arises from the amorphous nature of the as-FGSB film increasing the noise, while the annealed films were polycrystalline, such that the long range order helped to reduce the measured noise. The change from amorphous to crystalline structure within the films occurred between the annealing temperatures $100{ }^{\circ} \mathrm{C}$ and $250{ }^{\circ} \mathrm{C}$, which is in good agreement with Aboaf et al. [15] who determined that $\mathrm{FeSi}$ amorphous films became crystallised between the annealing temperatures of $180{ }^{\circ} \mathrm{C}$ and $250{ }^{\circ} \mathrm{C}$. From Bragg's law the lattice constants were determined and given in Table 2. For all the annealed films, the lattice constant is the same $2.89 \AA$, suggesting that the annealing temperature does not change the stress within the films. Previous work [8] on FeSiB films, found that $\alpha$ $\mathrm{Fe}$ crystallites formed due to annealing. The lattice constant of $\mathrm{Fe}$ is $2.87 \AA$ and for FeSiB is $2.868 \AA$, both of which are smaller than the lattice constant measured in these FeGaSiB films. FeSi has nine different phases [23] depending on the $\mathrm{Si}$ concentration and the temperature. The cubic stable phases at $300 \mathrm{~K}$ are BCC $\mathrm{Fe}_{3} \mathrm{Si}(a=5.64 \AA)$, cubic cFeSi $(a=2.77 \AA)$ and cubic $\varepsilon$-FeSi $(a=4.48 \AA)$. Therefore, although there is an increase in the $\mathrm{Si}$ content within the films, no additional phases such as $\mathrm{Fe}_{3} \mathrm{Si}\left(2 \theta=45.53^{\circ}\right)$ are observed, meaning that the polycrystalline structure is still Fe-BCC. The FeGaSiB lattice constant increase could be due to homogenous strain in the films due to the $\mathrm{Ga}$ and $\mathrm{Si}$ atoms being present.

The broad peak at $\sim 56^{\circ}$ with the additional sharper peaks are from the silicon substrate, ( $\mathrm{Si}$ (311) peak), as these measurements were done by glancing angle. To achieve a large enough SNR for the film peaks, the glancing angle had to be large enough to go through the whole film, and in some cases the top of the silicon substrate as well as.

Fig. 2b-e show the AFM images of the FeGaSiB films, it is observed that the film annealed at $100{ }^{\circ} \mathrm{C}$ shows no grains and the surface roughness is $<1 \mathrm{~nm}$ (Table 2). As the annealing temperature is increased, so the grain size is increased. The 400 -FGSB film had a smaller grain size than the 350-FGSB film, probably due to the additional Si within the film.

From Fig. 3, it is observed that there are changes in the magnetisation hysteresis loops as the annealing temperature is increased. The as-FGSB and 100-FGSB films both had uniaxial anisotropy, while the 350-FGSB and 400-FGSB films were both isotropic. The 250-FGSB film hysteresis loops suggest there are two different magnetic phases within the film. From the AFM data (Fig. 2c) the 250-FGSB film has grains, within an amorphous matrix, which would lead to two switching fields in the hysteresis loop along the hard axis. For the coercive field (Fig. 3f), it is observed that $H_{c}$ linearly increases as the annealing temperature increases from $100{ }^{\circ} \mathrm{C}$. This is expected, as the films become more polycrystalline with increase in annealing temperature and therefore the magnetocrystalline anisotropy energy will increase, plus the grains will increase the number of domain wall pinning sites within the film, also increasing the $H_{c}$. For the as-FGSB and 100-FGSB films, the coercive field was taken from the hard axis loop. As the coercive field increased, so did the saturation field with annealing temperature.

Fig. 4 shows how the different magnetic properties change as a function of annealing temperature. The saturation induction $\left(\mu_{o} M_{\mathrm{s}}\right)$ decreased as the annealing temperature increased, with a drop of $\sim 40 \%$ between the 100-FGSB film and the 250-FGSB film. There are two reasons for this, the first is that the concentration of $\mathrm{Fe}$ in the film decreases with increasing annealing temperature and second is that the films become more crystalline when annealed at the higher temperatures. For example, the amorphous 100-FGSB film saturation induction was $1.27 \mathrm{~T}$ compared to $0.8 \mathrm{~T}$ for the polycrystalline 350-FGSB film, this is a $\sim 40 \%$ reduction in saturation induction for a 3 conc $\%$ reduction in Fe concentration, with a 5 conc\% increase in Si and a 2 conc $\%$ reduction in $\mathrm{B}$. The large difference will not solely be due to the small decrease in $\mathrm{Fe}$, rather it is likely to be due the morphology change from amorphous to crystalline. Aboaf et al. [15] showed that for $300 \mathrm{~nm} \mathrm{FeSi}$ thin films, the amorphous films had a higher saturation magnetisation compared to the crystallised films, for the same film composition.

The saturation induction of the amorphous 100-FGSB film is comparable to previous FeGaSiB films fabricated using the co-sputter-evaporation system $[13,14]$. The previous FGSB films' saturation induction were measured in the range $0.96 \mathrm{~T}-1.24 \mathrm{~T}$. The composition of these films were similar to the 100-FGSB film, with the Fe concentration be $\sim 82 \%$, while the ratio of Ga:Si:B were slightly different. This suggests that for amorphous FeGaSiB films, the Fe concentration dominates the saturation induction. It also means that annealing at $100{ }^{\circ} \mathrm{C}$ does not strongly change the magnetic properties.

The saturation induction of the 250-FGSB film and the 350-FGSB film were $0.77 \mathrm{~T}$ and $0.8 \mathrm{~T}$ respectively, both these films had the same composition within error (table 1) and were polycrystalline, thus showing consistency within the films for composition and morphology. For the 400-FGSB film, the induction was lower than the 350-FGSB film, which is likely to be due to additional $\mathrm{Si}$ within this film.

Using Eq. (1) the Curie Temperature for each of the films was determined (Fig. 4). It was found that the Curie Temperatures were all much lower than expected. For the as-FGSB film, the $T_{c}$ was $354{ }^{\circ} \mathrm{C}$, which was nearly $150{ }^{\circ} \mathrm{C}$ higher than all the other annealed films. It should be noted that the values determined are probably lower than the actual values, as the model has a very sharp drop off at $T_{c}$, while in general the change in magnetisation with temperature is more gradual. For comparison, the Curie Temperature of $\mathrm{FeSiB}$ is $418{ }^{\circ} \mathrm{C}$ [24], thus the addition of $\mathrm{Ga}$ has lowered the $T_{c}$ by $\sim 50{ }^{\circ} \mathrm{C}$, with a further $150{ }^{\circ} \mathrm{C}$ reduction due to annealing and the addition of $\mathrm{Si}$ from the substrate.

From Fig. 4, it is also observed that the magnetostriction constant decreases with annealing temperature. The as-FGSB film had the largest magnetostriction constant (29 ppm), this is due to the largest saturation induction and the film being amorphous. Previous work on FeGaB [25] and FeSi [15] thin films found that the magnetostriction constant of the films increased going from polycrystalline to amorphous with the addition of either B or Si. The amorphous nature of magnetic films seem to enhance the magnetostriction constant, compared to the polycrystalline structure. The 100-FGSB film magnetostriction constant was $17 \mathrm{ppm}$, which again is comparable with those studied previously [13,14], thus backing up the suggestion that an annealing temperature of $100{ }^{\circ} \mathrm{C}$ does not change the magnetic properties.

For the polycrystalline films (250-FGSB, 350-FGSB and 400-FGSB), the magnetostriction constants $(\sim 10 \mathrm{ppm})$ were lower than the amorphous films, this could be due to the internal stresses within the films 

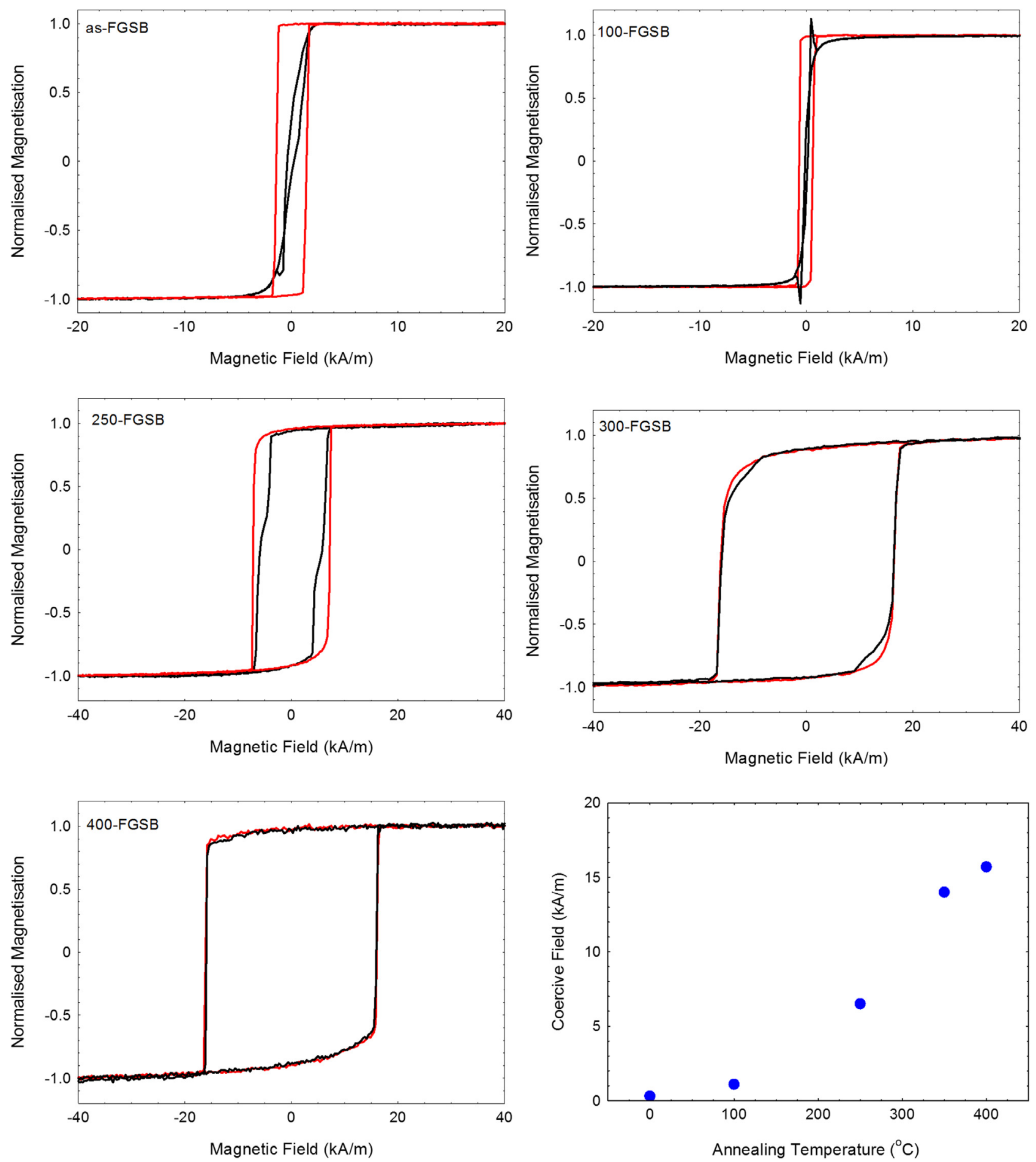

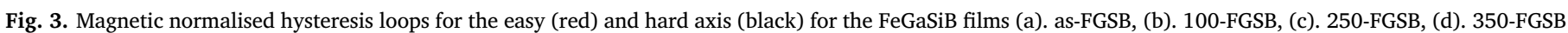

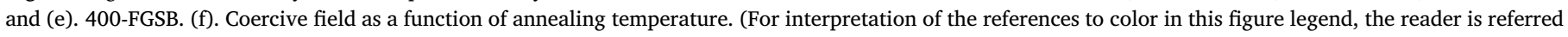
to the web version of this article.) .

decreasing the magnetostriction constant. From previous work, the magnetostriction constant of a magnetic film depends upon either the internal stresses within the film [26] or the surface/interface magnetostriction dominating [27]. The internal stresses arise during the fabrication process and the post-annealing process. The internal stresses can be due to the lattice mismatch or the thermal expansion between the film and the substrate. Also, it was found that for films with high internal stresses, the magnetostriction constants were lower than expected. An estimate of the internal stress within the annealed polycrystalline films can be determine from the XRD data. From Hooke's law the internal stress $\sigma$ in the plane, is linked to the $\varepsilon$ is the internal strain, given by $\varepsilon=\frac{a-a_{0}}{a_{0}}$ by [28]:

$\varepsilon=\frac{a-a_{o}}{a_{o}}=\frac{\left(1+\nu_{f}\right)}{Y_{f}} \sin ^{2} \psi \sigma-\frac{2 \nu_{f}}{Y_{f}} \sigma$

Where $Y_{f}$ is the Young's Modulus of the film, $\nu_{f}$ is the Poisson ratio of the film and $\psi$ is the tilt angle of the sample. For the films measured in this paper, $\psi=0$, thus the biaxial stress is given by $\sigma=-\frac{Y_{f}}{2 v_{f}} \varepsilon$. Taking the 


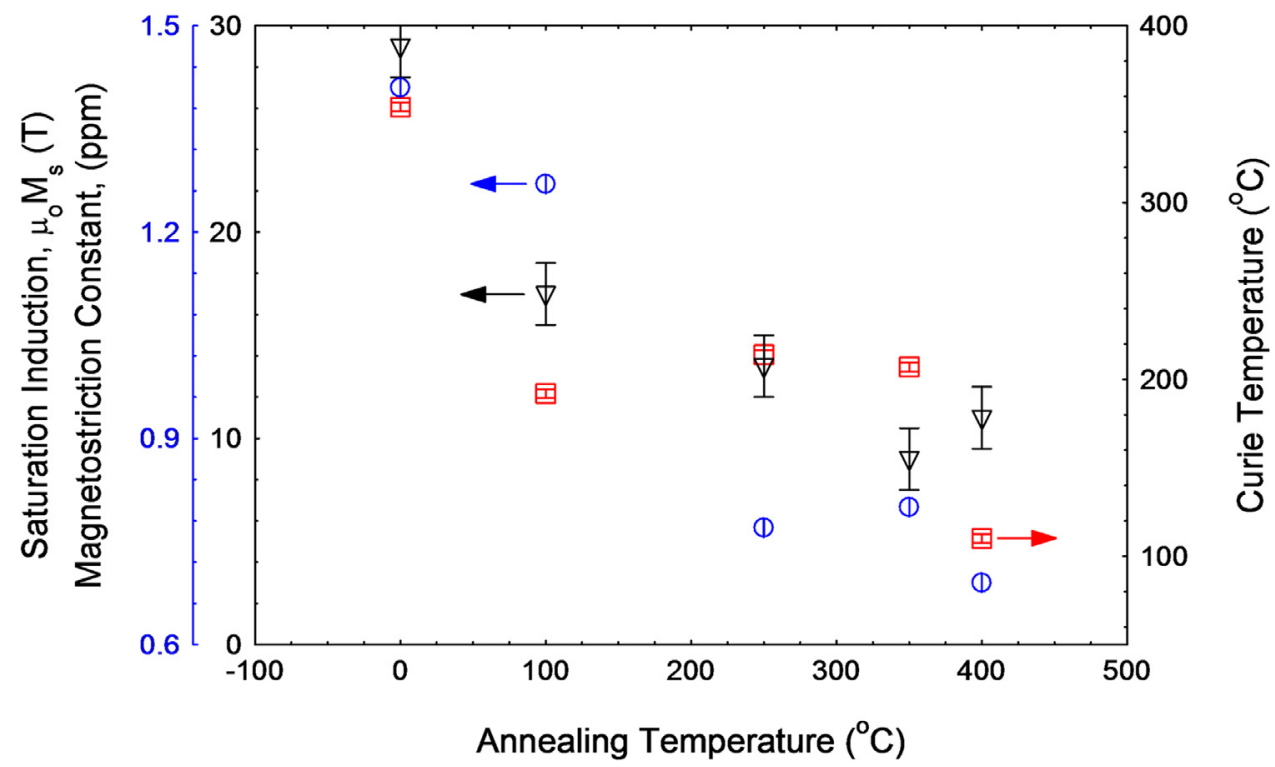

Fig. 4. Saturation Induction (blue circles), Curie Temperature (red squares), and Magnetostriction constant (black triangles) as a function of annealing temperature. (For interpretation of the references to color in this figure legend, the reader is referred to the web version of this article.).

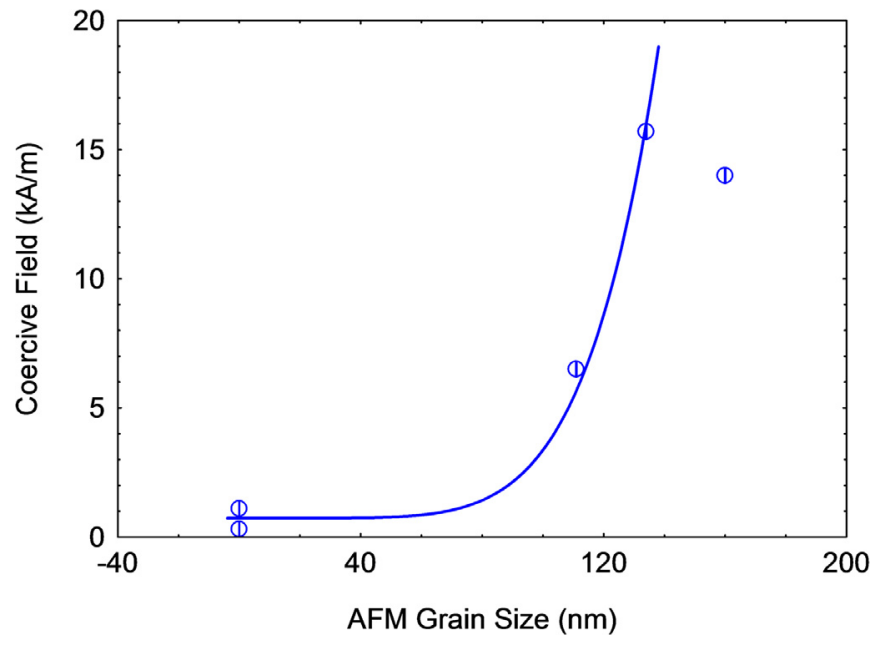

Fig. 5. Coercive field as a function of grain size. The solid line is a fit to the data.

Young's modulus of FeGaSiB films to be $120 \mathrm{GPa}$ [29], the Poisson ratio to be 0.28 and the strain determined from the lattice constant $(a)$ measured with respect to the FeSiB lattice constant $\left(a_{o}\right)$. This gives an internal stress of $\sim-1.85 \mathrm{GPa}$, which is comparable to internal stresses measured for other magnetostrictive films [16,27], where the magnetostriction constant was lower than expected. Although annealing is expected to reduce the stress within the films, in these films the stress is still large. This could be due to the short annealing time, being long enough for crystallisation to occur, but not long enough for equilibrium to be reached within the crystal structure.

From Herzer work [6], the behaviour of soft magnetic materials can be predicted from their grain size. The paper predicts that the coercive field increases as $D^{6}$ up to the grain size being roughly the same as the exchange length, and then decreases as $1 / D$ as the grain size continues to increase. From Fig. 5, it is observed there is a sharp increase in the coercive field, as the grain size increases in the films. The solid line is a $D^{6}$ fit to the data. At a grain size greater than $135 \mathrm{~nm}$, the coercive field starts to decrease with grain size. A linear fit is not included, as only two points are plotted in this region.

There are two exchange lengths quoted in the literature associated with the Bloch Wall (bulk) and the Neel Wall (thin film) [7]. The exchange length associated with the magnetocrystalline anisotropy and Bloch walls is given by:

$L_{e x}=\sqrt{\frac{A}{K_{1}}}$

Where $A$ is the exchange stiffness, for an amorphous material this is normally between 1 and $2 \times 10^{-11} \mathrm{~J} / \mathrm{m}$ and $K_{1}$ is the anisotropy constant of the material. As FeGaSiB is a new material, the anisotropy constant has not be determined for it. Coisson et al. [30] determine the in-plane anisotropy constant of FeSiB thin films to be $\sim 2500 \mathrm{~J} / \mathrm{m}^{3}$, taking these values the exchange length for an amorphous material based on $\mathrm{FeSiB}$ is $\sim 63-90 \mathrm{~nm}$.

While the exchange length associated with Neel walls and the magnetostatic anisotropy is:

$l_{e x}=\sqrt{\frac{2 A}{\mu_{o} M_{s}^{2}}}$

where $A$ is the exchange stiffness, and $M_{s}$ the saturation magnetisation. Substituting the $A$ and $M_{s}$ for the films into Eq. (6) gives $l_{e x}$ to be $10 \mathrm{~nm}$. Whether Bloch walls or Neel walls or even cross-tie walls occur within a thin magnetic film, depends upon the film thickness, along with the magnetisation and anisotropy constant [31]. A rough estimate of where this occurs is when the film thickness $\sim L_{e x}$, which is the case for the FeGaSiB films studied. Schneider et al. [32] also showed that $60 \mathrm{~nm} \mathrm{Fe}$ films contained Bloch walls using magnetic force microscopy. Thus the FeGaSiB films studied here are on the Neel-Bloch wall borderline. As Herzer work assumes Bloch walls, this means that the films are on the boundary to fit $\mathrm{D}^{6}$ to the data, but from Fig. 5, the FeGaSiB films show the expected behaviour of soft magnetic materials, with the coercive field dependence on the grain size switching at roughly the Bloch wall exchange length.

\section{Conclusions}

Annealing the FeGaSiB thin films changed the film composition, along with both the structural and magnetic properties of the films. Annealing above $250{ }^{\circ} \mathrm{C}$ caused the $\mathrm{Si}$ in the substrate to diffuse across the interface into the films, but no additional FeSi phases were formed. The higher annealing temperatures also caused the films to become crystalline, with the surface roughness and grain size increasing with 
increasing annealing temperature. Similarly, the coercive field increased with increasing annealing temperature, which was linked to the increase in grain size. As the films became polycrystalline with the higher annealing temperatures, so the saturation induction decreased, which also led to a decrease in the Curie Temperature and magnetostriction constant. Thus the structural transition from amorphous to polycrystalline strongly changes the magnetic properties of the FeGaSiB films.

\section{Contribution breakdown}

Writing: NAM (100\%)

Experimental: MR (25\%), QA (25\%), SB (10\%), SG (10\%), NAM $(30 \%)$

Figures: NAM (100\%)

Analysis: MR (33\%) NAM (67\%)

\section{Declaration of Competing Interests}

The authors declare that they have no known competing financial interests or personal relationships that could have appeared to influence the work reported in this paper.

\section{Acknowledgements}

XPS data collection was performed at the EPSRC National Facility for XPS ('HarwellXPS'), operated by Cardiff University and UCL, under contract No. PR16195. Funding: This work was supported by the Royal Society Mid-Career Leverhulme Trust Fellowship scheme (SRF $\backslash R 1 \backslash 180020)$ and the Leverhulme Trust (RPG-2018-324).

\section{Reference}

[1] M.R.J. Gibbs, E.W. Hill, P.J. Wright, Magnetic materials for MEMS applications, J. Phys. D Appl. Phys. 37 (22) (2004) R237-R244, https://doi.org/10.1088/00223727/37/22/R01.

[2] O.F. Hikmat, M.S.M. Ali, RF MEMS inductors and their applications - a review, J. Microelectromech. Syst. 26 (1) (2017) 17-44, https://doi.org/10.1109/JMEMS. 2016.2627039.

[3] A.L. Herrera-May, J.C. Soler-Balcazar, H. Vazquez-Leal, J. Martinez-Castillo, M.O. Wigueras-Zuniga, L.A. Aguilera-Cortes, Recent advances of MEMS resonantors for Lorentz force based magnetic field sensors: design, applications and challenges, Sensors 16 (2016) 1359, https://doi.org/10.3390/s16091359.

[4] C.O Mathuna, N. Wang, S. Kulkarni, S. Roy, Review of integrated magnetics for power supply on chip (PwrSoC), IEEE Trans. Power Electron. 27 (11) (2012) 4799-4816, https://doi.org/10.1109/TPEL.2012.2198891.

[5] D. Viehland, M. Wuttig, J. McCord, E. Quandt, Magnetoelectric magnetic field sensors, MRS Bull. 43 (11) (2018) 834-840, https://doi.org/10.1557/mrs.2018. 261.

[6] G. Herzer, Grain size dependence of coercivity and permeability in nanocrystalline ferrromagnets, IEEE Trans. Magn. 26 (5) (1990) 1397-1402, https://doi.org/10. $1109 / 20.104389$.

[7] G.S. Abo, Y-K. Hong, J. Park, J. Lee, W. Lee, B-C. Choi, Definition of magnetic exchange length, IEEE Trans. Magn. 49 (8) (2013) 4937-4939, https://doi.org/10. 1109/TMAG.2013.2258028.

[8] Z.G. Sun, H. Kuramochi, M. Mizuguchi, F. Takano, Y. Semba, H. Akinaga, Magnetic properties and domain structures of FeSiB thin films, Surf. Sci. 556 (2004) 33-38, https://doi.org/10.1016/j.susc.2004.02.036.

[9] M. Neagu, M. Dobromir, G. Popa, H. Chiriac, G. Singurel, C. Hison, The surface magnetism investigation of $\mathrm{FeSiB}$ amorphous thin films obtained by evaporation technique, Sens. Actuators A 129 (2006) 172-175, https://doi.org/10.1016/j.sna. 2005.11.037.
[10] A Javed, T Szumiata, N A Morley, M R J Gibbs, An investigation of the effect of structural order on magnetostriction and magnetic behaviour of $\mathrm{Fe}-\mathrm{Ga}$ alloy thin films, Acta Mater. 58 (2010) 4003-4011, https://doi.org/10.1016/j.actamat.2010. 03.023 .

[11] N A Morley, S- L Yeh, S Rigby, A Javed, M R J Gibbs, Development of a cosputterevaporation for Fe-Ga films, J. Vac. Sci. Technol., A 26 (4) (2008) 581 586.10.1116/1.2924416.

[12] J.R. Hattrick-Simpers, D. Hunter, C.M. Craciunescu, K.S. Jang, M. Murakami, J. Cullen, M. Wuttig, I. Takeuchi, S.E. Lofland, L. Benderksy, N. Woo, R.B.V. Dover, T. Takahashi, Y. Furuya, Combinatorial investigation of magnetostriction in Fe-Ga and Fe-Ga-Al, Appl. Phys. Lett. 93 (2008) 102507, , https://doi.org/10.1063/1. 2980034.

[13] Q.A. Abbas, N.A. Morley, Fabrication and characterisation of magnetostrictive amorphous FeGASiB thin films, J. Magn. Magn. Mater. 439 (2017) 353-357, https://doi.org/10.1016/j.jmmm.2017.04.097.

[14] Q.A. Abbas, N.A. Morley, A. Johansson, T. Thomson, Influence of Ar pressure on the magnetic properties of amorphous FeGaSiB thin films, IEEE Trans. Magn. 53 (11) (2017) 2502504, , https://doi.org/10.1109/TMAG.2017.2696059.

[15] J.A. Aboaf, R.J. Kobliska, E. Klokholm, Properties of transition metal-metalloid ferromagnetic thin films, IEEE Trans. Magn. 14 (5) (1978) 941-943, https://doi. org/10.1109/TMAG.1978.1059923.

[16] A. Caruana Finkel, N. Reeves-McLaren, N.A. Morley, Influence of soft magnetic underlayers on the magnetic properties of $\mathrm{Co}_{90} \mathrm{Fe}_{10}$ films, J. Magn. Magn. Mater. 357 (2014) 87-92, https://doi.org/10.1016/j.jmmm.2014.01.030.

[17] N.W. Ashcroft, N.D. Mermin, Solid State Physics, Sauders College, Philadephia, 1976.

[18] T.J. Broomhall, A.W. Rushforth, M.C. Rosamond, E.H. Linfield, T.J. Hayward, Suppression of dynamically-induced stochastic magnetic behaviour through materials engineering, Phys. Rev. Appl. 13 (2) (2020) 024039.

[19] A. Javed, N.A. Morley, M.R.J. Gibbs, Structure, magnetic and magnetostrictive properties of as-deposited Fe-Ga thin films, J. Magn. Magn. Mater. 321 (2008) 2877-2882, https://doi.org/10.1016/j.jmmm.2009.04.039.

[20] N.A. Morley, D. Dhandapani, A. Rao, H. Al Qahtani, M.R.J. Gibbs, H. Grell, D. Eastwood, B.K. Tanner, Polymeric spin-valves at room temperature, Synth. Method 161 (2011) 558-562, https://doi.org/10.1016/j.synthmet.2010.11.011.

[21] T. Isobe, H. Nakashima, K. Hashimoto, Diffusion coefficient of interstitial iron in silicon, Jpn. J. Appl. Phys. 2 (7) (1989) 1282-1283, https://doi.org/10.1143/JJAP. 28.1282.

[22] A. Gupta, D. Kumar, V. Phatak, Asymmetric diffusion at the interfaces in $\mathrm{Fe} / \mathrm{Si}$ multilayers, Phys. Rev. B 81 (2010) 155402, , https://doi.org/10.1103/PhysRevB. 81.155402.

[23] R. Nakane, S. Sugahara, M. Tanaka, Structural and magnetic properties of ferromagnetic $\mathrm{Fe}_{1-\mathrm{x}} \mathrm{Si}_{\mathrm{x}}(0.18<\mathrm{x}<0.33)$ films formed by rapid thermal annealing on silicon-on insulator substrates, J. Appl. Phys. 117 (2015) 133906, , https://doi.org/ 10.1063/1.4915335.

[24] J.M. Borrego, J.S. Blazquz, C.F. Conde, A. Conde, S. Roth, Structural ordering and magnetic properties of arc-melted FeGa alloys, Intermetallics 15 (2007) 193-200, https://doi.org/10.1016/j.intermet.2006.05.007.

[25] J. Lou, R.E. Insignares, Z. Cai, K.S. Ziemer, M. Liu, N.X. Sun, Soft magnetism, magnetostriction and microwave properties of FeGaB thin films, Appl. Phys. Lett. 91 (2007) 182504, , https://doi.org/10.1063/1.2804123.

[26] E. du Tremolet, de Lacheisserie, J.C. Peuzin, Magnetostriction and internal stresses in thin films: the cantilever method revisited, J. Magn. Magn. Mater. 136 (1994) 189-196, https://doi.org/10.1016/0304-8853(94)90464-2.

[27] N.A. Morley, S. Rigby, M.R.J. Gibbs, Anisotropy and magnetostriction constants of nanostructured $\mathrm{Fe}_{50} \mathrm{Co}_{50}$ films, J. Optoelectron. Adv. Mater. 12 (2009) 109-113.

[28] F. Motazedian, Z Wu, J. Zhang, B.S. Shariat, D. Jiang, M. Martyniuk, Y. Liu, $\mathrm{H}$. Yang, Determining intrinic stress and strin state of fibre-textured thin films by Xray diffraction measurements using combined asymmetrical and Bragg-Brentano configurations, Mater. Des. 181 (2019) 108063, , https://doi.org/10.1016/j. matdes.2019.108063.

[29] Q.A. Abbas, Magnetic and Mechanical Properties of Magnetostrictive FeGaSiB Films, PhD Thesis, Univ. Sheff. (2018).

[30] M. Coisson, C. Appino, F. Celegato, A. Magni, P. Tiberto, F. Vinai, Magnetisation process in sputtered FeSiB thin films, Phys. Rev. B 77 (2008) 214404, , https://doi. org/10.1103/PhysRevB.77.214404.

[31] S. Middelhoek, Domain walls in thin Ni-Fe films, J. Appl. Phys. 34 (1963) 1054.10.1063/1.1729367.

[32] M. Schneider, St. Muller-Pfeifer, W. Zinn, Magnetic force microscopy of domain wall fine structures in iron films, J. Appl. Phys. 79 (1996) 8578-8583, https://doi. org/10.1063/1.362539. 